\title{
Relações entre a atenção primária e as internações por condições sensíveis em um hospital universitário
}

\author{
Relations between the primary care and hospitalizations due to sensitive conditions \\ in a university hospital \\ Relaciones entre la atención primaria y las internaciones por condiciones sensibles \\ en un hospital universitario
}

\section{Lucia Aparecida de Souza ${ }^{a, b}$ \\ Ricardo de Mattos Russo Rafael ${ }^{a, c}$ \\ Anna Tereza Miranda Soares de Moura ${ }^{a, d}$ \\ Mercedes Neto ${ }^{c}$}

\section{Como citar este artigo:}

Souza LA, Rafael RMR, Moura ATMS, Neto M. Relações entre a atenção primária e as internaç̄ões por condiç̧es sensíveis em um hospital universitário. Rev Gaúcha Enferm. 2018;39:e2017-0067. doi: https:// doi.org/10.1590/1983-1447.2018.20170067
- Universidade Estácio de Sá, Programa de Pós-Graduação em Saúde da Família. Rio de Janeiro, Rio de Janeiro, Brasil.

Universidade Federal de Juiz de Fora (UFJF), Hospita Universitário. Juiz de Fora, Minas Gerais, Brasil.

'Universidade do Estado do Rio de Janeiro (UERJ), Faculdade de Enfermagem, Departamento de Enfermagem de Saúde Pública. Rio de Janeiro, Rio de Janeiro, Brasil.

Universidade do Estado do Rio de Janeiro (UERJ), Faculdade de Medicina, Departamento de Pediatria. Rio de Janeiro, Rio de Janeiro, Brasil.

\section{RESUMO}

Objetivo: Analisar as relações entre a presença e a orientação da Atenção Primária à Saúde e as internações por condições sensíveis à atenção primária em um hospital universitário.

Método: Estudo seccional com amostra de 197 sujeitos internados no período de março a junho de 2016 na clínica médica de um hospital universitário de Juiz de Fora. A aferição, realizada por meio de entrevistas, utilizou o Primary Health Care Assessment Tool, versão reduzida para adultos, e a lista brasileira de condições sensíveis, editada pelo Ministério da Saúde.

Resultados: A frequência regular à Atenção Primária esteve associada às internações (RP:2,06), especialmente frente ao baixo desempenho dos atributos de acesso (RP:5,3) e da integralidade (RP:4,7).

Conclusão: A baixa orientação dos atributos da Atenção Primária sugere que somente a cobertura deste nível talvez não seja suficiente para reduzir estas internações, mas a forma que ela se organiza e efetiva no nível comunitário.

Palavras-chave: Avaliação em saúde. Atenção primária à saúde. Hospitalização. Hospitais universitários. Adulto.

\section{ABSTRACT}

Objective: To analyze the relations between the presence and orientation of the Primary Health Care and hospitalizations due to sensitive conditions to primary care in a university hospital.

Method: This is a sectional study with a sample of 197 subjects hospitalized from March to June 2016 in the medical clinic of a university hospital in Juiz de Fora. The assessment, conducted through interviews, used the Primary Health Care Assessment Tool, reduced version for adults, and the Brazilian list of sensitive conditions, edited by the Ministry of Health.

Results: The regular frequency of the Primary Care was associated with hospitalizations (OR:2,06), especially related to the low performance of the access attributes (OR:5,3) and the comprehensives (OR:4,7).

Conclusion: The low orientation of the Primary Care attributes suggests that only the coverage at this level may not be sufficient to reduce these hospitalizations, but the way it is organized and effective at the community level.

Keywords: Health evaluation. Primary healthcare. Hospitalization. Hospitals, university. Adult.

\section{RESUMEN}

Objetivo: Analizar las relaciones entre la presencia y la orientación de la Atención Primaria de la Salud y las internaciones por condiciones sensibles a la atención primaria en un hospital universitario.

Método: Estudio seccional con una muestra de 197 pacientes hospitalizados en el período de marzo a junio 2016 en el clínica médica de un hospital universitario de Juiz de Fora. Los evaluación, realizada a través de entrevistas, utilizada lo Primary Health Care Assessment Tool, versión reducida para adultos, y la lista brasileña de condiciones sensibles, editada por el Ministerio de la Salud. Resultados: La frecuencia regular de la Atención Primaria se asoció con hospitalizaciones (RP:2,06), especialmente frente al bajo desempeño de los atributos de acceso (RP:5,3) y de integralidad (RP: 4,7).

Conclusión: La orientación baja de los atributos de la Atención Primaria sugieren que solamente la cobertura de este nivel tal vez no sea suficiente para reducir estas internaciones, sin embargo, la forma que ella se organiza y se efectiva a nivel comunitario. Palabras clave: Evaluación en salud. Atención primaria de salud. Hospitalización. Hospitales universitários. Adulto. 


\section{Q INTRODUÇÃO}

A Atenção Primária à Saúde (APS) apresenta múltiplas concepções que podem estar presentes - de modo isolado ou conjugado - em um mesmo modelo de sistema de saúde ${ }^{(1)}$. Estas concepções classificam a APS como: seletiva, de primeiro nível da atenção ou como abrangente. A primeira prima por programas restritos e focalizados em populações carentes ou em cestas de serviço voltadas ao controle de doenças prevalentes. A segunda prevê a APS como porta de entrada do sistema e, até certo ponto, também está direcionada ao atendimento das principais doenças da população. A última concepção, a APS abrangente ou integral, também entendida como estratégia, pressupõe a (re)organização do sistema por meio de uma abordagem que compreende a saúde como direito humano, sendo atravessada por reflexões de ordem social, tais como o entendimento que o saneamento, a alimentação, o trabalho são fenômenos que também permeiam os cuidados deste nível da atenção(2).

No Brasil, a APS vem se estruturando conforme as reformas histórico-sociais do sistema de saúde. Também têm sido pautadas no atendimento às múltiplas realidades locais e, portanto, não têm se desenvolvido de modo linear e na mesma proporção em todo território nacional. Tendo em vista a pluralidade cultural, as diferenças políticogeográficas e as diferenças socioeconômicas das regiões brasileiras, a APS tem se mostrado como um conjunto de ações que guardam certa plasticidade na conformação do trabalho, sempre guiada pelos princípios da multiprofissionalidade e territorialização(1).

O modelo de APS adotado pelo Sistema Único de Saúde (SUS) brasileiro prima pela responsabilização das ações de promoção, prevenção, tratamento, reabilitação e redução de danos, voltados às necessidades individuais e coletivas de agregados humanos, e tendo como missões a coordenação do cuidado e o ordenamento da rede. Espera-se que esta APS seja norteada por princípios de acessibilidade, vínculo como instrumento terapêutico e de gestão, da continuidade e integralidade do cuidado, e da co-responsabilização do usuário, metas ainda desafiadoras pela diversidade de cenários que compõem o país e pela necessidade de maior suporte financeiro e político pelo setor público ${ }^{(2-3)}$.

Esta modelagem de APS está alicerçada em sete atributos, sendo quatro essenciais - acesso do primeiro contato, longitudinalidade, integralidade e coordenação da atenção - e três derivados - orientação centrada na família, orientação comunitária e competência cultural, este último considerado como sendo de difícil avaliação. Com o intuito de ampliar a qualidade da APS e reconhecer seus limites e suas possibilidades de atuação, muito se tem produzido sobre a avaliação em saúde neste campo. A identificação das fragilidades dos serviços e a geração de subsídios para o planejamento das ações têm sido frequentemente recomendadas pelo Ministério da Saúde, utilizando um instrumental internacionalmente conhecido: o Primary Care Assessment Tool - PCAToo (3-4) . $^{(2)}$

Outra possibilidade de avaliação que vem sendo frequentemente realizada é a investigação das Internações por Condições Sensíveis à Atenção Primária (ICSAP) como forma de monitoramento sistemático da APS e da Rede de Atenção. Alguns autores afirmam que um número elevado destas internações podem ser evitadas com ações efetivas da APS ${ }^{(5)}$. Elevadas taxas de ICSAP podem caracterizar faIhas operacionais no sistema, seja por barreiras de acesso ou pela qualidade de assistência prestada.

No entanto, parece imprescindível apontar que ainda é parca a produção de estudos que conjugam estratégias de avaliação destes constructos. Observa-se que, em geral, realizam-se inferências sobre a qualidade das ações baseadas em um destes atributos, o que pode estar gerando perdas significativas de componentes analíticos e, até certo ponto, da real associação entre a exposição - referente à presença da APS - e o desfecho - a redução da ICSAP.

Maior dificuldade se tem em associar esta análise a um ambiente de produção acadêmica - de ensino, pesquisa e extensão - aliada à prestação de assistência nas 24 horas diárias: os hospitais universitários; que desde a sua concepção foram alvo de disputas de modelos assistenciais e formadores. De um lado, a crença da formação biomédica e centrada na especialidade. De outro, um ambiente de criação, de alta densidade tecnológica leve-dura e dura, e parte integrante de uma complexa rede assistencial, fato este que estaria integrado à formação dos novos profissionais de saúde(6).

Tomando como referência o desafio cotidiano da construção de uma rede ampliada que contemple estes hospitais enquanto pontos de atenção integrados, tem-se como questão de pesquisa: o desempenho da APS reduz as internações por condições sensíveis nestas unidades? Compreendendo que uma das formas de aferição do desempenho deste nível está na forma como são desenvolvidos os atributos específicos e derivados, este estudo tem por objetivo analisar as relações entre a presença e a orientação da Atenção Primária à Saúde e as internações por condições sensíveis à atenção primária em um hospital universitário. Espera-se, com isso, contribuir para o debate sobre avaliação da rede a partir de elementos presentes no cotidiano destes equipamentos: as internações e a dinâmica de funcionamento da APS. 


\section{MÉTODOS}

Trata-se de estudo transversal realizado na unidade de clínica médica do hospital universitário de Juiz de Fora. 0 hospital é centro de referência em atendimento de usuários da rede do SUS do município e regiões, exercendo atividades no nível secundário, constituído de ambulatórios especializados e terciário, composto por seis setores de internação. Com total de 140 leitos de enfermarias, alcança uma média mensal de 300 internações.

A população do estudo foi composta por pacientes internados na unidade de clínica médica do hospital universitário no período de março a junho de 2016. Os critérios de inclusão do estudo foram: o tempo de internação superior a 24 horas, conforme padronização da nomenclatura do censo hospitalar ${ }^{(7)}$ e idade igual ou superior a 18 anos. Foram excluídos os pacientes que se encontravam impossibilitados de participação por limitações clínicas, como as situações de desorientação e sedação, avaliadas sistematicamente pela primeira autora do estudo.

Para o cálculo do tamanho amostral foi considerada a média de internações mensais de 100 pacientes, a prevalência estimada de 0,35, o intervalo de confiança de 95\% e o nível de precisão de 5\%, perfazendo a amostra mínima de 187 sujeitos. Considerando a dificuldade associadas aos inquéritos hospitalares, foi realizada uma sobreamostra de 10 sujeitos, perfazendo o tamanho amostral final de 197 sujeitos.

A seleção dos participantes ocorreu com base na lista de internações das últimas 48 horas, fornecida pela central de internação hospitalar. O convite de participação ocorreu somente após aplicar os critérios de inclusão, sendo sempre necessária a consulta ao prontuário para avaliação de possíveis limitações cognitivas. A coleta de dados foi realizada pela primeira autora deste estudo, por meio de abordagem à beira do leito, em dias alternados e no horário vespertino, conciliando com os horários de visitas e evitando interrupções das entrevistas.

Foi utilizado questionário multidimensional contendo instrumentos validados e adaptados transculturalmente para uso no Brasil, conforme descrito em cada dimensão. A primeira dimensão foi composta por itens que avaliaram o perfil sociodemográfico, tais como idade, sexo, tempo de estudo, situação conjugal e moradia. A avalição da classe econômica, pertencente a esta dimensão, ocorreu com base nos Critérios de Classificação Econômica da Associação Brasileira de Empresas de Pesquisa( ${ }^{(8)}$. Na segunda dimensão estiveram presentes as situações de acompanhamento dos serviços de saúde ${ }^{(9)}$. Destaca-se que as duas primeiras dimensões capturaram possíveis variáveis confundidoras para o modelo final de análise. Na terceira dimensão buscou-se aferir as variáveis independentes do estudo, ou seja, os atributos da APS: afiliação (frequência regular à APS para promoção dos cuidados), acesso de primeiro contato (acesso e utilização do serviço pelo usuário), longitudinalidade (atributo que apresenta a relação contínua dos profissionais com os usuários, ou seja, um mesmo profissional acompanha o paciente nas suas condições específicas de saúde através do tempo), coordenação (atributo que responde pela viabilização da continuidade na assistência por meio da integração dos serviços de saúde), integralidade (atributo que se caracteriza pela capacidade de organização e articulação da atenção em rede) e orientação familiar (capacidade de reconhecimento da importância do contexto e da dinâmica da relação social familiar no processo saúde-doença) e comunitária (reconhecimento das necessidades de saúde da comunidade e, por meio de tal conhecimento, o estabelecimento dos planos assistenciais).

A terceira dimensão foi aferida com o instrumento Primary Care Assessment Tool (PCATool-Brasil), versão adulto, em sua forma reduzida e validada para uso no Brasil( ${ }^{(10)}$. 0 instrumento, que é composto por 23 itens do tipo Likert, com quatro opções de respostas e valores variaram de 1 (com certeza, não) a 4 (com certeza, sim), passando por "provavelmente sim" e "provavelmente não". As respostas "não sei/não lembro" não foram consideradas para fins de cálculo, conforme orienta o manual do instrumento(4).

O cálculo dos escores foi obtido pela média aritmética simples do total de itens de cada escala (atributo), conforme recomendado pelo manual de utilização do instrumento ${ }^{(4)}$. O escore geral, que avalia a orientação geral da APS, dada pela associação de todos os atributos, foi calculado a partir do escore médio de cada escala, incluindo o grau de afiliação, e dividido pelo total de componentes. Já o cálculo do escore essencial, que responde pela associação dos atributos essenciais da APS (acesso de primeiro contato, longitudinalidade, coordenação e integralidade) ocorreu a partir do mesmo método do escore anterior, exceto por considerar apenas o grau de afiliação, o acesso de primeiro contato, a longitudinalidade, a coordenação e a integralidade. Em todos os casos houve a conversão dos valores em escala que variou de zero, a menor orientação do atributo de cada escala, a 10, a maior orientação. Seguindo o "Manual do Instrumento de Avaliação da Atenção Primária à Saúde", adotou-se o ponto de corte de 6,66 pontos para aceitar a classificação de "forte grau/nível de orientação" de cada atributo(4).

A última dimensão do questionário buscou identificar o tipo de internação de cada participante, variável dependente do estudo, classificando como ICSAP e não-ICSAP a partir da lista brasileira editada pelo Ministério da Saúde(11). 
O software Microsoft Excel 2010 foi utilizado para a construção do banco de dados. O processamento estatístico ocorreu no software Stata SE 13. A análise bivariada constou de cálculo das razões de prevalência (RP) bruta e seus respectivos intervalos de confiança a 95\%, utilizando o Teste Exato de Fisher e os valores menores que 0,05 como estatisticamente significantes e limítrofes quando no intervalo entre 0,05 e 0,1. Destaca-se que o ponto de corte de 0,25 foi adotado para a entrada no modelo de regressão não-condicional, utilizado para o ajustamento das RP. A fim de testar as possíveis interações, aplicou-se a técnica de análise logística condicional, utilizando como variável independente de interesse aquela que apresentou valor inferior a 0,05 na estatística bivariada. Aplicou-se o Teste de Razão de Verossimilhança como teste de hipótese nos modelos logísticos.

Visando cumprir os preceitos éticos previstos pela Resolução CNS no 466/2012, o projeto de pesquisa foi submetido e aprovado pelo Comitê de Ética sob o no 1.427.653, e também utilizou Termos de Consentimento Livre e Esclarecido antes da coleta de dados. Além disso, todos os entrevistados foram avaliados clinicamente antes e após o inquérito, a fim de observar alterações psicobiológicas, tais como alterações pressóricas, grau de orientação e aspectos específicos da internação. Não houve registro de intercorrências, mas cabe informar que equipe multiprofissional (médicos, enfermeiros, técnicos de enfermagem) monitorou os procedimentos de entrevista como forma de suporte clínico da pesquisa.

\section{RESULTADOS}

Das 400 internações esperadas para o período, ocorreram somente 269. Destas, 42 (15,6\%) usuários não atenderam os critérios de seleção, 13 (4,8\%) já haviam participado do estudo em internação anterior, 13 (4,8\%) indivíduos recusaram, e apenas quatro $(1,5 \%)$ tiveram alta antes da coleta de dados, sendo classificados como perdas de seguimento.

As principais características sociodemográficas da amostra estudada se concentraram em mulheres (53,8\%), na faixa etária entre 18 e 59 anos $(62,9 \%)$, casada (50,0\%), com mais de quatro anos de estudo (80,7\%), pertencente à classe econômica C (57,9\%) e residentes da zona urbana de Juiz de Fora $(69,5 \%)$. A prevalência de ICSAP na população estudada foi de $20,8 \%(n=41)$.

A Tabela 1 apresenta a análise bivariada entre as internações e as características sociodemográficas.

Tabela 1 - Análise bivariada das Condições Sensíveis à Atenção Primária em função das características sociodemográficas. Juiz de Fora, Minas Gerais, Brasil. 2016. ( $n=197)$

\begin{tabular}{|c|c|c|}
\hline Variáveis sociodemográficas & RP (IC 95\%) & p-valor \\
\hline \multicolumn{3}{|l|}{ Sexo } \\
\hline Feminino & 1 & \\
\hline Masculino & $1,3(0,6-2,6)$ & 0,469 \\
\hline \multicolumn{3}{|l|}{ Faixa etária } \\
\hline De 18 a 59 anos & 1 & 0,077 \\
\hline 60 anos e mais & $1,2(1,0-1,4)$ & \\
\hline \multicolumn{3}{|l|}{ Etnia } \\
\hline Brancos/Amarelos/Indígena & 1 & \\
\hline Pretos e pardos & $1,6(0,8-3,1)$ & 0,208 \\
\hline \multicolumn{3}{|l|}{ Tempo de estudo } \\
\hline Mais de quatro anos & 1 & \\
\hline Menos de quatro anos & $0,9(0,5-1,6)$ & 0,817 \\
\hline \multicolumn{3}{|l|}{ Situação conjugal } \\
\hline Outros & 1 & \\
\hline Casados / União estável & $0,6(0,3-1,3)$ & 0,221 \\
\hline \multicolumn{3}{|l|}{ Classe econômica } \\
\hline Classe A/B & 1 & \\
\hline Classe C & $0,9(0,3-3,1)$ & 0,930 \\
\hline Classe D/E & $1,1(0,3-3,7)$ & 0,939 \\
\hline \multicolumn{3}{|l|}{ Zona urbana } \\
\hline Sim & 1 & \\
\hline Não & $1,2(0,6-2,6)$ & 0,564 \\
\hline
\end{tabular}

Fonte: Dados da pesquisa, 2016.

Legenda: RP- Razão de Prevalência; IC- Intervalo de Confiança; p- valor do teste Exato de Fisher. 
Na Tabela 1 foi observado que somente a faixa etária foi estatisticamente significante de modo limítrofe, mesmo apresentando RP próximo de 1,0. Em continuidade à apresentação da análise bivariada do estudo, a Tabela 2 apresenta a associação entre as internações por condições sensíveis à Atenção Primária e as variáveis de acompanhamento de saúde dos pacientes.
Na Tabela 2 chama-se atenção para a associação, mesmo após o modelo de ajustamento, entre a presença da APS (RP: 2,6; IC95\%: 1,3/5,5) e as internações por condições sensíveis.

A Tabela 3 apresenta a análise bi e multivariada entre o baixo escore geral (orientação da APS) e essencial (atributos essenciais), bem como a baixa orientação dos demais atributos da APS e as ICSAP.

Tabela 2 - Análise bivariada e de regressão logística das Condições Sensíveis à Atenção Primária e as variáveis de acompanhamento de saúde. Juiz de Fora, Minas Gerais, Brasil. 2016. ( $n=197)$

\section{Variáveis}

RP bruta (IC 95\%)

RP ajustada (IC95\%)

\section{Primeiro serviço que buscou antes da internação}

$\begin{array}{lcc}\text { APS } & 1 & 1 \\ \text { Outros } & 1,1(0,4-2,8) & 0,9(0,3-2,5) \\ \text { p-valor } & 0,905 & 0,869\end{array}$

\section{Serviço que referenciou}

APS

Outros

p-valor

\section{Opção de internar no hospital}

Já realiza acompanhamento ou teve indicação no hospital

Vaga regulada

p-valor

Tipo de unidade no bairro

ESF

Outros

p-valor

\section{Presença da APS (frequenta regularmente)}

Não

Sim

p-valor

1
$1,3(0,1-11,7)$
0,800

1

$0,6(0,3-1,3)$

$$
\begin{gathered}
1,3(0,1-11,5) \\
0,835
\end{gathered}
$$

$0,5(0,3-1,1)$

0,092

Fonte: Dados da pesquisa, 2016.

Legenda: RP - Razão de Prevalência; RP ajustada por faixa etária, escolaridade, situação conjugal e tipo de unidade no bairro; IC - Intervalo de Confiança; APS - Atenção Primária à Saúde; ESF - Estratégia Saúde da Família; p-valores do teste Exato de Fisher e de Razão de Verossimilhança.

Tabela 3 - Análise bivariada e de regressão logística das Condições Sensíveis à Atenção Primária em função da percepção sobre a extensão e a orientação da APS. Juiz de Fora, Minas Gerais, Brasil. 2016. (n=197)

\section{Variáveis sociodemográficas}

RP bruto (IC95\%)

RP ajustada (IC95\%)

\section{Acesso de primeiro contato}

Alto grau

Baixo grau

p-valor

$1,1(0,6-1,7)$
0,793

$1,0(0,6-1,7)$

0,925

\section{Longitudinalidade}

Alto grau

Baixo grau

p-valor

\section{1}

$1,1(0,5-2,2)$

0,814

1

$0,9(0,3-2,5)$

0,905
1

$1,1(0,5-2,3)$

0,820

1

$1,0(0,3-2,6)$

0,953 


\section{Coordenação}

Alto grau

Baixo grau

p-valor

\section{Integralidade}

Alto grau

Baixo grau

p-valor

\section{Orientação familiar}

Alto grau

Baixo grau

p-valor

\section{Orientação comunitária}

\author{
Alto grau \\ Baixo grau \\ p-valor
}

\section{Escore Essencial}

Alto grau

Baixo grau

p-valor

\section{Escore Geral}

Alto grau

Baixo grau

p-valor

$$
\begin{gathered}
1,0(0,4-2,4) \\
0,912
\end{gathered}
$$

1

$1,0(0,5-2,1)$

0,966

1

$1,3(0,6-2,7)$

0,458

1

$1,8(0,9-3,7)$

0,083

$1,3(0,6-2,8)$

0,551

1

$1,0(0,4-2,1)$

0,936

$1,2(0,5-2,8)$

0,722

$1,0(0,5-2,1)$

0,983

1

$1,3(0,6-2,8)$

0,551

1

1

$2,1(1,1-4,4)$ 0,044

1

$1,2(0,6-2,5)$

0,621
$1,0(0,4-2,3)$

Fonte: Dados da pesquisa, 2016.

Legenda: RP - Razão de Prevalência; RP ajustada por faixa etária, escolaridade, situação conjugal; p-valores do teste Exato de Fisher e de Razão de Verossimilhança.

Destaca-se que o modelo ajustado aponta para relação estatisticamente significante apenas em relação ao escore essencial (RP: 2,$1 ; 1,1 / 4,4)$. Por fim, a Tabela 4 demonstra, por técnica de regressão logística condicional, a relação entre a presença da APS e as ICSAP em função da baixa orientação dos atributos.

Tabela 4 - Análise logística condicional da presença da Atenção Primária em função da baixa extensão dos atributos. de

\begin{tabular}{|c|c|c|}
\hline Baixa orientação do... & RP (IC95\%) & p-valor \\
\hline Escore Essencial (Atributos essenciais) & $3,8(1,4-10,6)$ & 0,010 \\
\hline Escore Geral (Orientação da APS) & $3,6(1,3-10,5)$ & 0,017 \\
\hline Acesso de primeiro contato & $5,3(1,7-16,6)$ & 0,005 \\
\hline Longitudinalidade & $3,4(1,3-8,5)$ & 0,011 \\
\hline Coordenação & $3,2(1,2-8,5)$ & 0,018 \\
\hline Integralidade & $4,7(1,6-13,5)$ & 0,004 \\
\hline Orientação familiar & $3,3(1,2-8,9)$ & 0,020 \\
\hline Orientação comunitária & $2,3(0,8-9,2)$ & 0,106 \\
\hline
\end{tabular}
Juiz de Fora, Minas Gerais, Brasil. 2016. $(n=197)$

Fonte: Dados da pesquisa, 2016.

Legenda: RP - Razão de Prevalência ajustada por faixa etária, escolaridade, situação conjugal e tipo de

unidade no bairro; APS - Atenção Primária à Saúde; p-valor do Teste de Razão de Verossimilhança.

Destaca-se que os valores referenciais se constituem em relação à "forte orientação" dos atributos. Chama-se atenção para a interação em todos os modelos, exceto quando envolvida orientação comunitária. 


\section{口 DISCUSSÃO}

O estudo apontou para associação entre a presença de cuidados prestados pela APS e as maiores chances de ICSAP (RP: 2,$7 ; 1,2 / 5,9)$ no hospital, coadunando com pesquisas realizadas no Paraná(12) e no Distrito Federal(13). O menor de potencial de resolutividade das unidades de saúde, a baixa cobertura da Estratégia Saúde da Família e, como consequência, a maior fragilidade de saúde dos sujeitos são apontadas como possíveis elementos que permeiam este fenômeno de hospitalizações evitáveis.

Por outro lado, a maior parte dos estudos ecológicos realizados, refere à presença da APS como fator associado à exponencial redução das internações por condições sensíveis ${ }^{(14-15)}$. E, do lado oposto, grupos de pesquisa também trazem a informação sobre a inobservância destas relações, refletindo que o indicador de ICSAP, por si, não é suficiente para avaliar a APS(16).

Mesmo com a variedade de posições e os múltiplos percursos metodológicos adotados nas investigações, existe forte corrente que aponta que a redução das hospitalizações está associada à qualidade das ações da APS e não meramente à presença dela. Na tentativa de analisar o impacto da APS na atenção terciária, alguns autores associaram a avaliação de desempenho à tipologia das internações ${ }^{(17)}$. Nesse ponto, cabe marcar posição que as ICSAP, por quase a totalidade dos autores, são compreendidas como eventos complexos e evitáveis, dependendo do modo como a APS atua no território(14-15,17).

Acredita-se que, até certo ponto, a redução das hospitalizações esteja atrelada à eficiência e à resolutividade deste nível de atenção e, sobretudo, da sua capacidade de articulação com os demais pontos da Rede. Entende-se que esta hipótese ganha força nos achados deste estudo, especialmente no que tange a elevação das chances de internações face ao baixo grau de orientação nos atributos essenciais da APS - o acesso, a integralidade, a longitudinalidade e a coordenação do cuidado -, o que reforça a ideia de que a dinâmica de atuação das equipes pode estar relacionada ao evento.

A necessidade de melhoria na configuração dos serviços de APS se eleva na medida em que as ICSAP estão frequentemente associadas às doenças crônicas, que, como já exposto por diversos autores, estão associadas às dificuldades de acesso e de adesão do usuário ao tratamento(18). Ressalta-se que as ICSAP trazem prejuízos tanto do ponto de vista do sistema de saúde, uma vez que aumenta os gastos públicos, como para o indivíduo e para a sociedade, acarretando em danos psicobiológicos, psicossociais e de ordem econômica ${ }^{(5)}$.

O sistema de saúde que se orienta por uma APS seletiva e/ou como porta de entrada não tem impacto na redução das hospitalizações, uma vez que não mobilizam os neces- sários recursos de base territorial e sistêmica para enfrentar os problemas de saúde da população(19). Ou seja, acabam por concentrar energia de trabalho e conhecimentos para resolver, em última análise, estritamente as doenças da população. Embora o Ministério da Saúde tenha adotado a APS com estratégia de ordenamento dos sistemas, em alguns serviços, é perceptível a presença de uma APS pouco potente e de baixa orientação.

Ressalta-se que a coordenação do cuidado, atributo que dispõe sobre a viabilização da continuidade na assistência por meio da integração dos serviços de saúde ${ }^{(20)}$, requer a inclusão e participação ativa de todos os pontos de atenção envolvidos no processo de cuidado. A falta de sistemas efetivamente organizados de referência e contra-referência parecem ampliar às internações desnecessárias em nível especializado, especialmente pela visão estritamente biomédica que os serviços de APS seletiva tendem a desenvolver.

As fragilidades de articulação das Redes somadas à disputa dos modelos entre a APS - que deveria ser abrangente - e os serviços de média e de alta complexidade pode guardar influências de ordem macroestrutural e não captadas neste estudo. A formação de vínculo entre os serviços especializados e os usuários também figura entre os aspectos que podem permear as relações de hospitalização, já que os hospitais universitários atraem pacientes em busca de resolutividade dos problemas. Somam-se a isto as dificuldades também próprias dessas instituições, que invariavelmente enfrentam problemas políticos e de inclusão nas de Redes de Atenção, gerando dificuldades de retorno dos pacientes aos seus serviços de origem.

É importante considerar que os dados aqui produzidos devem ser interpretados à luz de algumas limitações. O primeiro limite, e talvez o principal, está no percurso metodológico assumido para esta investigação. Estudos transversais aferem a exposição e o desfecho simultaneamente, o que, pela falta de temporalidade entre os eventos, inviabiliza estabelecer os nexos de causalidade. Portanto, os achados aqui expostos devem ser interpretados tão somente como hipóteses explicativas, mesmo com indiscutível relevância à produção de conhecimento. Outro aspecto que merece destaque é que a apreciação dos atributos da APS e das ICSAP, a partir dos pacientes internados, pode conter viés de seleção super ou subestimando os dados a partir da variação da exposição entre os indivíduos, especialmente pela ausência de ambientes controlados para a alocação dos grupos de usuários em relação ao tipo das internações.

\section{- CONCLUSÕES}

A presença de cuidados prestados pela APS esteve associada a maiores chances de ICSAP, especialmente frente ao baixo grau de orientação dos atributos essenciais. Ao 
que parece, não basta a implementação de ações deste nível. O modo como a APS exerce seu papel parece influenciar diretamente sobre as hospitalizações evitáveis. Deste modo, pensar em resolutividade da APS é também refletir sobre a necessária inter-relação dos atributos que a constitui e também sobre a posição que ocupa enquanto ordenadora da Rede de Atenção.

Mesmo frente a estas limitações, é possível refletir que a disponibilidade de leitos no hospital universitário conjugada com problemas nos processos regulatórios de vagas esteja influenciando este quadro de hospitalizações, que a rigor deveriam ser evitadas no primeiro nível da atenção. A literatura aponta que ainda é persistente - até nas equipes multiprofissionais - a concepção de que o hospital é o melhor espaço para a implementação de condutas terapêuticas e diagnósticas, sobretudo quando se trata de local extremamente especializado e voltado ao ensino e à pesquisa, como no caso do cenário de estudo. Tal pensamento, norteado pelo desejo de melhor tratar, pode estar aumentando os encaminhamentos destes pacientes pelos profissionais da APS. Outro aspecto é o vínculo já estabelecido entre usuários e os ambulatórios especializados, que também pode trazer pressões para que estes encaminhamentos sejam realizados. Deste modo, a cultura biomédica e da hospitalização pode estar ganhando força frente às fragilidades na organização de algumas equipes de APS, aumentando as chances de ICSAP.

No limite do estudo é possível observar a presença e a organização dos cuidados prestados pela APS a partir da visão dos indivíduos internados em um hospital universitário. Sendo assim, parece indispensável reafirmar que a opção metodológica deste estudo não possibilita a investigação de aspectos de ordem macroestrutural do sistema, fato esse que estimula o desenvolvimento de novas produções que associem mais fatores ao modelo analítico das ICSAP e dos atributos de desempenho da APS.

\section{口EFERÊNCIAS}

1. Mendes EV. O Cuidado das condições crônicas na atenção primária à saúde: imperativo da consolidação da estratégia da saúde da família. Brasília: Organização Pan-Americana da Saúde; 2012.

2. Giovanella L, Mendonça MHM. Atenção primária à saúde. In: Giovanella L, Escorel S, Lobato LVC, Noronha JC, Carvalho AI (organizadores). Políticas e sistemas de saúde no Brasil. 2. ed. Rio de Janeiro: Fiocruz; 2012. p. 493-577.

3. Macinko J, Harris MJ. Brazil's family health strategy: delivering community-based primary care in a universal health system. N Engl J Med. 2015;372(23):2177-81.

\section{- Autor correspondente:}

Ricardo de Mattos Russo Rafael

E-mail: prof.ricardomattos@gmail.com
4. Ministério da Saúde (BR). Manual do instrumento de avaliação da atenção primária à saúde. Brasília: Ministério da Saúde; 2010.

5. Alfradique ME, Bonolo PF, Dourado I, Lima-Costa MF, Macinko J, Mendonça CS, et al. Internações por condições sensíveis à atenção primária: a construção da lista brasileira como ferramenta para medir o desempenho do sistema de saúde (Projeto ICSAP - Brasil). Cad Saúde Pública. 2009;25(6):1337-49.

6. Freund T, Campbell SM, Geissler S, Kunz CU, Mahler C, Peters-Klimm, et al. Strategies for reducing potentially avoidable hospitalizations for ambulatory caresensitive conditions. Ann Fam Med. 2013:11(4):363-70.

7. Ministério da Saúde (BR). Padronização da nomenclatura do censo hospitalar. 2. ed. rev. Brasilia: Ministério da Saúde; 2002.

8. Associação Brasileira de Empresas de Pesquisa. Critério de classificação econômica Brasil. São Paulo: ABEP; 2014.

9. Ferrer APC. Avaliação da atenção primária à saúde prestada a crianças e adolescentes na região oeste do município de São Paulo [tese]. São Paulo (SP): Faculdade de Medicina, Universidade de São Paulo; 2013.

10. Oliveira MMC, Harzheim E, Riboldi J, Duncan BB. PCATool-ADULTO-BRASIL: uma versão reduzida. Rev Bras Med Fam Comunidade. 2013;8(29):256-63.

11. Ministério da Saúde (BR). Portaria n. 221, de 17 de abril de 2008: dispõe sobre a Lista Brasileira de Internações por Condições Sensíveis à Atenção Primária. Brasília; 2008 [citado 2016 dez 17]. Disponível em: http://bvsms.saude.gov.br/ bvs/saudelegis/sas/2008/prt0221_17_04_2008.html.

12. Petilin EB, Gutubir D, Molena-Fernandes CA, Pelloso SM. Sensitive female-specific hospitalization in primary care. Ciênc Saúde Coletiva. 2015;20(2):441-8.

13. Sousa NP, Rehem TCMSB, Santos WS, Santos CE. Hospitalizations sensitive to primary health care at a regional hospital in the Federal District. Rev Bras Enferm. 2016; 69(1):118-25.

14. Muraro CF, Gigante LP, Nedel FB, Carvalho TGML, Domenech SC, Gevaerd MS. [The family health strategy in relation to hospitalization for primary care sensitive conditions in elderly]. Rev Baiana Saúde Pública. 2013:37(1):20-33. Portuguese.

15. Brasil VP, Costa JSD. Hospitalizations owing to ambulatory care sensitive conditions in Florianopolis, Santa Catarina, Brazil:- an ecological study, 2001-2011. Epidemiol Serv Saúde. 2016;25(1):75-84.

16. Costa JP, Jorge MSB, Vasconcelos MGF, Paula ML, Bezerra IC. Resolubilidade do cuidado na atenção primária: articulação multiprofissional e rede de serviços. Saúde Debate. 2014;38(103):733-43.

17. Oliveira VB, Turci MA, Lima FF, Bonolo PF, Macinko J, Wong LLR, et al. Avaliação do impacto das ações do Programa de Saúde da Família na redução das internações hospitalares por condições sensíveis à Atenção Básica em adultos e idosos. Belo Horizonte: Núcleo de Educação em Saúde Coletiva (NESCON); 2012.

18. Santos VCF, Kalsing A, Ruiz ENF, Roese A, Gerhardt TE. A profile of admittances to hospital due to non-contagious chronic diseases sensitive to primary health care among chronologically advantaged patients in the southern half of Rio Grande do Sul. Rev Gaúcha Enferm. 2013;34(3):124-31.

19. Rehem TCMSB, Ciosak SI, Egry EY. Ambulatory care sensitive conditions: general hospital of micro-region of São Paulo municipality, Brazil. Texto Contexto Enferm. 2012;21(3):535-42.

20. Oliveira MAC, Pereira IC. [Primary Health Care essential attributes and the Family Health Strategy]. Rev Bras Enferm. 2013;66(esp):158-64. Portuguese. 